Article

\title{
A Simplified Physical Model Construction Method and Gas-Water Micro Scale Flow Simulation in Tight Sandstone Gas Reservoirs
}

\author{
Fengjiao Wang ${ }^{1}{ }^{(\mathbb{D})}$, Yikun Liu ${ }^{1, *}$, Chaoyang Hu ${ }^{1, *}$, Anqi Shen ${ }^{1}{ }^{(D)}$, Shuang Liang ${ }^{1}$ and Bo Cai ${ }^{2}$ \\ 1 Department of Petroleum Engineering, Northeast Petroleum University, Daqing 163318, China; \\ wangfengjiao8699@126.com (F.W.); anqi1986@126.com (A.S.); liangshuang21@163.com (S.L.) \\ 2 Petrochina Exploration and Development Research Institute at Langfang, Langfang 065007, China; \\ caibo69@petrochina.com.cn \\ * Correspondence: shenliujili@163.com (Y.L.); huchaoyang8699@126.com (C.H.)
}

Received: 22 May 2018; Accepted: 11 June 2018; Published: 14 June 2018

\begin{abstract}
Accuracy defects exist when modeling fluid transport by the classical capillary bundle model for tight porous media. In this study, a three-dimensional simplified physical model construction method was developed for tight sandstone gas reservoirs based on the geological origin, sedimentary compaction and clay mineral-cementation. The idea was to reduce the porosity of the tangent spheres physical model considering the synergistic effect of the above two factors and achieve a simplified model with the same flow ability as the actual tight core. Regarding the wall surface of the simplified physical model as the boundary and using the Lattice Boltzmann (LB) method, the relative permeability curves of gas and water in the simplified model were fitted with experimental results and a synergistic coefficient could be obtained, which we propose for characterizing the synergistic effect of sedimentary compaction and clay mineral-cementation. The simplified physical model and the results simulated by the LB method are verified with the experimental results under indoor experimental conditions, and the two are consistent. Finally, we have carried out a simulation of gas flooding water under conditions of high temperature and high pressure which are consistent with the actual tight sandstone gas reservoir. The simulation results show that both gas and water have relatively stronger seepage ability compared with the results of laboratory experiments. Moreover, the interfacial tension between gas and water is lower, and the swept volume is larger during placement. In addition, the binding ability of the rock surface to the water film adhered to it becomes reduced. The method proposed in this study could indicate high frequency change of pores and throats and used to reflect the seepage resistance caused by frequent collisions with the wall in microscopic numerical simulations of tight sandstone gas reservoirs.
\end{abstract}

Keywords: tight sandstone gas reservoir; simplified physical model; Lattice Boltzmann method; percolation mechanism; micro-scale

\section{Introduction}

Classical capillary bundle models rely heavily on extensions of Darcy's law and empirical relationships that do not comprehensively capture all of the important physical phenomena at various scales [1-3]. For instance, the common approach used to study two-phase of gas and water or gas alone systems in subsurface environments is to consider each phase separately using Darcy's law and then account for the characteristics of physical parameters by classical capillary bundle models [4-6]. This approach can't explicitly accounted for the multidirectional transport of gas molecules and the variation of the seepage resistance caused by the higher frequency change of the flow radius on the micro-scale for tight sandstone gas reservoirs. Additionally, it can't be ignored that the rock surface 
has a definitive influence on the fluid flow laws [7-10]. This suggests that in addition to the factors considered by the capillary bundle models, a physical model that accounts for the micro-scale effect and the multiple pore-scale fluid configurations must be set up.

Many achievements have also been made in the study of pore-throat structure from a microscopic scale viewpoint. Zhao et al. [11] applied the multidirectional scanning method to search pores and throats, and tried to set up a simplified model, but they failed to distinguish the locations of pores and throats exactly. Shin et al. [12] achieved the segmentation of pores and throats through the method of dividing the central axis in pores, however, their approach had poor generality due to the complex algorithm. Al-Kharusi et al. [13] presented a methodology for extracting networks from pore space images, while the properties of cores fitted with the indoor experiment results. Numerous authors have shown that Lattice Boltzmann models are able to simulate three-dimensional multiphase and multi-component fluid flows in complex porous systems [14-17]. Lei et al. [18] simulated the desorption and diffusion process of gas in porous media with complex structures by a $2 \mathrm{D}$ digital core based on CT scanning and combined the evolution equation of the LBM. Based on the cross-correlation-based simulation (CCSIM) combined with the three-step sample method, Ji et al. [19] reconstructed stochastic $3 \mathrm{D}$ models of the heterogeneous porous media, while problems existed in the boundary condition at the rock wall during the micro-scale simulation. From this brief review, it is clear that no definitive and concise physical models have been proposed to characterize the fluid flow on the micro-scale, and the corresponding numerical simulation method is also inadequate, thus further study is warranted.

Spencer [20] suggested that the ideal soil model consisted of sphere particles with the same diameter. In this case, the porosity was $47.6 \%$ and $25.96 \%$ by the loosest and the closest arrangement, respectively [21]. Supposed that there were two different sizes of rock particles, the larger particles were arrayed orthogonally while the smaller particles were exactly tangent to each larger one, thus the porosity was $12.5 \%$ in such cases. The statistical results demonstrated that the span range of the porosity in tight sandstone gas reservoirs was approximately $6 \%$ to $12 \%$ in China [22,23], which made it possible to set up simplified physical models for tight sandstone gas reservoirs based on the above arrangement mode. Accordingly, the objective of this study is to propose a simplified physical model construction method for tight sandstone gas reservoirs and conduct LB simulations of gas flooding water in a simplified physical model in order to investigate the percolation characteristics of gas and water at the micro-scale. In Section 2, the notion of a simplified physical model that is based on the geological origin of tight sandstone gas reservoirs and assumes that the sizes of sandstone particles follow the characteristics of a Rayleigh distribution is briefly described. In Section 3, we put forward the sphere model of particles tangent to each other, the sphere model of particles according with the Rayleigh frequency distribution and the sphere model of particles embedded under the synergistic effect of compaction and cementation. The change laws of the seepage cross-sectional areas in each element were calculated and presented. Subsequently, a synergistic coefficient was proposed to characterize the coupling effects of the above factors and its fitting method was also established by applying the LBM in Section 4. Finally, we presented the results and discussion regarding the difference and comparisons between the simulations and experimental results for the percolation mechanism of gas flooding water.

\section{The Construction Idea of the Simplified Model}

Rock particles are always poorly in sorted for tight sandstone gas reservoirs, and the pores and the throats are easily blocked by more tiny particles [24]. The tight sandstone gas reservoir characteristics and fluid properties of the Sulige Gasfield were taken as the research object. The grading analysis results demonstrated that permeability of most rock cores were not matched to the median sizes of the rock particles. This was due to the sedimentary compaction and clay mineral-cementation in tight sandstone gas reservoirs. Thus, the porosity and the permeability were generally lower (Figures 1 and 2). 


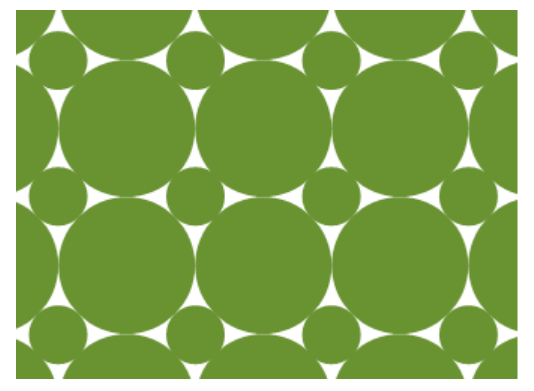

Figure 1. Tangent arrangement of rock particles without extrusion.

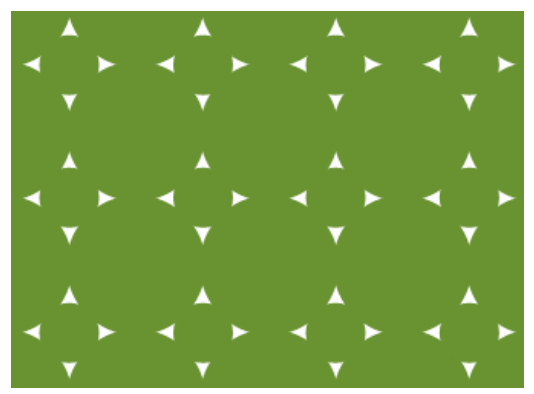

Figure 2. Tangent arrangement of rock particles under compaction.

The statistical data showed that the sizes of sandstone particles conformed to the characteristics of a Rayleigh frequency distribution [25]. In order to set up a three-dimensional simplified model of tight porous media based on its geometric structure, we implemented a physical model as the basic model where the particles of various sizes followed the Rayleigh frequency distribution in the longitudinal direction, and the spherical particles in the same layer were the same in size. What was more, the particles are tangent to each other. Due to the differences in the degree of mineral cementation, the permeability of different cores might be different even in a similar particle size frequency distribution. In view of the above situation, the sealing radius $r_{\mathrm{c}}$ of the clay minerals which was determined by the actual tight sandstone gas cores was introduced to characterize the simplified models. Subsequently, aiming at the effect of sedimentation-compaction under higher pressure conditions, the compaction coefficient was introduced to characterize the strength of compaction in tight gas reservoirs. Generally, the porosity and the seepage ability of the simplified physical model are equivalent to the actual tight cores; this simplified model can be applied to study the fluid percolation laws and other relevant researches. Therefore, LBM was applied to simulate gas and water flow and the simulation results were utilized to fit the relative permeability curves of gas and water in turn. Finally, the sealing radius and the compaction coefficient could be confirmed and the corresponding three-dimensional simplified model of a tight sandstone gas core could be formed.

It was considered that the particle sizes of the tight sandstone particles satisfied the Rayleigh frequency distribution as Equation (1):

$$
f(r)=\left\{\begin{array}{l}
\frac{r}{\sigma^{2}} e^{-\frac{r^{2}}{2 \sigma^{2}}}, r_{\min } \leq r \leq r_{\max } \\
0, \text { others }
\end{array}\right.
$$

In the simplified model, it is considered to be the fluid flowing space where the radius $r$ of the particles is in the range of $r_{\mathrm{c}}$ to $r_{\max }$. That is to say that this part is considered to be the fluid flow space in the LB model. It is considered to be the cemented position where the radius $r$ of the particles is in the range of $r_{\min }$ to $r_{\mathrm{c}}$.

Based on the method above, the pore space is reduced and the corresponding porosity of the simplified model is equivalent to the actual tight sandstone core. Under the conditions that the 
sandstone particle sizes are in the range of $r_{1}$ to $r_{2}$, the corresponding frequency distribution probability can be calculated by Equation (2):

$$
g\left(r_{1}, r_{2}\right)=\int_{r_{1}}^{r_{2}} f(r) \mathrm{d} r
$$

\section{Construction Method of the Simplified Model}

\subsection{The Tangent Spheres Model}

For the tight sandstone gas reservoirs, the alternating frequency of pores and throats are always very high and the collision probability between fluid and rock wall is greatly increased, resulting in higher seepage resistance. There's no doubt that accuracy defects exist for modeling fluid transport by the classical capillary bundle model. Consequently, we developed a simplified physical model for tight porous media in this study. At first, the tangent spheres model was proposed based on the following assumptions: in a cube with a side length of $4 R$, eight sphere particles with a radius of $R$ are tangent to each other and they are also tangent to the surface of the cube; a smaller sphere particle was in the middle of the cube, and it was tangent to the other eight large particles which was shown in Figure 3a; obviously, the radius of the smaller one was $(\sqrt{3}-1) R$ according to the geometric relation. The blue smaller cube in Figure $3 \mathrm{~b}$ was regarded as a percolation microelement, in which was the free flow space for fluid except the portion occupied by the sphere particles. In the blue cube, the percolation section-area were changed continuously as shown in Figure 4 which was similar to the alternation of pore and throat.

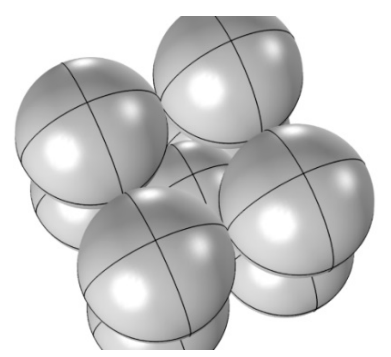

(a)

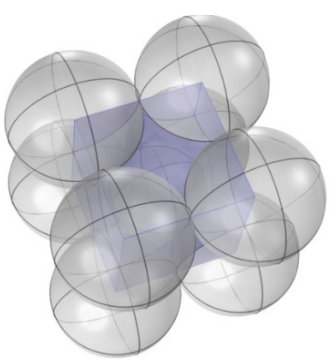

(b)

Figure 3. The microelement of the simplified three-dimensional physical model for the tight sandstone gas reservoirs. (a) A cube microelement in the physical model; (b) The blue cube is a percolation microelement.

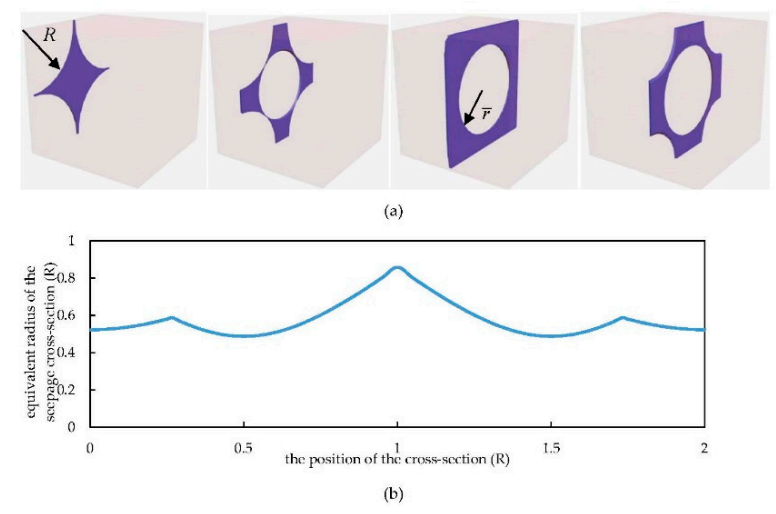

Figure 4. The changing process of the seepage cross-sectional area in a percolation microelement. (a) The continuously changing cross section represents the pore space of the blue area in Figure $3 \mathrm{~b}$.

(b) The equivalent radius at any position $\mathrm{x}$ in a percolation microelement. 
In order to obtain the mathematical relation between the radius of particle and the radius of flow space, suppose that the left cross section of the blue cube is at the position of $x=0, \bar{r}$ denotes the equivalent flow radius of any position $x$, and $r^{\prime}$ denotes the equivalent flow radius of the entire flow space when a percolation microelement is viewed as a tube with a length of $2 R$. According to the geometric relationship above, the equivalent radius at any position $\mathrm{x}$ of the flow space in a percolation microelement is as follows:

$$
\bar{r}= \begin{cases}\sqrt{\frac{4 R^{2}}{\pi}-\left(R^{2}-x^{2}\right)}, & x \in[0,(2-\sqrt{3}) R) \\ \sqrt{\frac{4 R^{2}}{\pi}-\left(R^{2}-x^{2}\right)-\left\{R^{2}-[x-(\sqrt{3}-1) R]^{2}\right\},} & x \in[(2-\sqrt{3}) R, R)\end{cases}
$$

Thus, if a percolation microelement is viewed as a tube with a length of $2 R$, the equivalent flow radius of the entire flow space can be expressed as:

$$
\pi \times\left(r^{\prime}\right)^{2} \times 2 R=\left(\int_{0}^{(2-\sqrt{3}) R} \pi \times \bar{r}^{2} d x+\int_{(2-\sqrt{3}) R}^{R} \pi \times \bar{r}^{2} d x\right) \times 2
$$

Substituting Equation (3) to Equation (4), we can obtain the approximate relationship between the equivalent radius and the sphere particle radius as Equation (5):

$$
r \approx 0.5796 R
$$

Equation (5) will be utilized to characterize the pore radius of the actual tight sandstone cores and the sizes of the sphere particles at the same scale in the subsequent numerical simulation.

As the tangent spheres model was composed of two different sizes of rock particles, definitely, its porosity and geometric shape were pretty much certain. In order to embody the distribution characteristics of the rock particle sizes in the actual tight reservoirs, we proposed the spheres model whose particle radius followed the Rayleigh frequency distribution.

\subsection{The Spheres Model Following the Rayleigh Frequency Distribution}

A large number of domestic and foreign research results have demonstrated that the physical parameters of sandstone reservoirs are quite different compared with the diagenetic period [26,27]. For tight sandstone gas reservoirs, the main reasons for that are sedimentary compaction and clay mineral-cementation [28,29]. Consequently, the simplified physical model was also developed based on the geological origin above.

The peak value $\sigma$ is the characteristic parameter of any Rayleigh distribution which determines the distribution function. Based on the actual distribution of rock particle radii $\mathrm{n}$ the tight sandstone gas reservoirs, we can obtain a deterministic distribution function and the value of $\sigma$ is also known. In the microelement, all center positions of the spheres remain unchanged and only the particle radius gets changed which follows the same distribution function in vertical. In the horizontal aspects, the sphere particles on the same layer are the same in radius. Thereby, we can obtain the spheres model following the Rayleigh distribution function, as is shown in Figure 5.

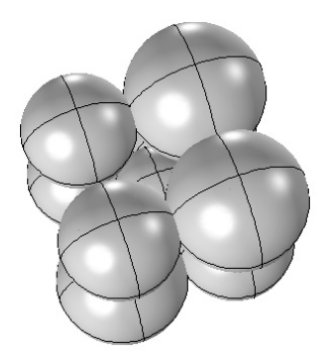

Figure 5. The spheres model following the Rayleigh distribution function in a microelement. 
Accordingly, there are embedded parts between particles, and there are also untouched parts between them due to the change in particle radius. Based on the geological origin of tight sandstone gas reservoirs: sedimentary compaction and clay mineral-cementation, we propose two ways of reducing the porosity for the tangent spheres physical model. For the embedded parts, it was considered to be caused by the sedimentary compaction. The untouched parts were considered to be cemented by minerals. However, it is unrealistic that all center positions of the spheres remain unchanged. In order to get the simplified physical model more consistent with the real tight cores, we propose the embedded spheres model under synergy effect of sedimentary compaction and clay mineral-cementation.

\subsection{The Embedded Spheres Model under the Synergy Effect of Compaction and Cementation}

Suppose that the sealing range of the cementation was just enough for the sphere with a radius of $r_{\mathrm{c}}$ to form the single point contact anywhere in the pore space. Under the premise of meeting the above conditions, as the sealing range reached the minimum, the corresponding radius was called "sealing radius of the cementation" and its diagram was shown in Figure 6. The sealing radius of the cementation could be calculated by the geometrical relationships of the particles in various sizes.

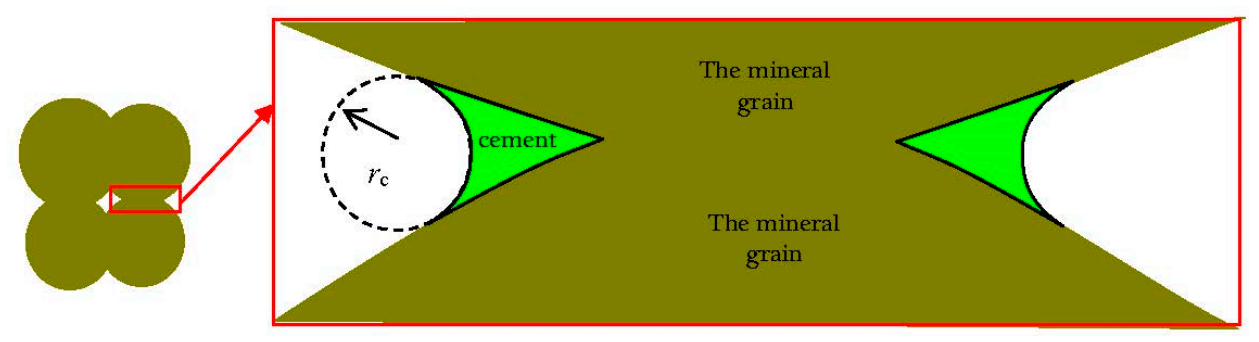

Figure 6. The diagram of the sealing range between two sizes of spherical particles.

The porosity of the embedded spheres model can be adjusted by changing the compaction strength and the sealing range of cementation. Namely, it could be obtained by decreasing the center positions between particles and changing the sealing radius of the cementation. We defined the porosity reduction value caused by the former as $\phi_{\mathrm{s}}$ and the later as $\phi_{\mathrm{c}}$, respectively. It is worth noting that the excessive compaction might lead to more narrow pores which would increase the bounding effect of the rock surface to the fluid. Conversely, if the sealing radius of cementation was too large, it would bring much smooth channels so that the simplified model could not accurately describe the complicated seepage flow space in tight porous media. Therefore, we proposed a synergistic coefficient $\alpha_{\mathrm{s}}$ to describe the ratio of the decreased porosity by the compaction to the total variation of the porosity, as was shown in Equation (6):

$$
\alpha_{\mathrm{s}}=\phi_{\mathrm{s}} /\left(\phi_{\mathrm{s}}+\phi_{\mathrm{c}}\right)
$$

Subsequently, for a core from tight sandstone gas reservoir, we suppose that the particle sizes distribution function and the porosity were already known, so we can take the same percolation capacity as the goal and fit the corresponding synergy coefficient $\alpha_{\mathrm{s}}$ of the simplified model. Thus, the simplified physical model which is equivalent to the actual tight gas core could be obtained.

\section{The Fitting Method of the Synergetic Coefficient}

In this study, the tight sandstone gas reservoir characteristics and fluid property of the Sulige Gasfield were taken as the research platform. The particle sizes' Rayleigh distribution function of each core could be obtained by carrying out the particle size analysis of tight cores. The basic parameters of each tight sandstone core are shown in Table 1. 
Table 1. The basic parameters of each tight sandstone core.

\begin{tabular}{ccccccc}
\hline Number & Depth $(\mathbf{m})$ & $\begin{array}{c}\text { Porosity } \\
\mathbf{( \% )}\end{array}$ & $\begin{array}{c}\text { Permeability } \\
\left(\times \mathbf{1 0}^{-\mathbf{3}} \boldsymbol{\mu m}^{\mathbf{2}}\right)\end{array}$ & $\begin{array}{c}\text { The Median Value of } \\
\text { the Pore Throat } \\
\text { Radius (nm) }\end{array}$ & $\begin{array}{c}\text { Smaller Sandstone } \\
\text { Particles } \sigma(\mathbf{n m})\end{array}$ & $\begin{array}{c}\text { The Fitting Value of } \\
\text { Rayleigh } \\
\text { Distribution } \sigma\end{array}$ \\
\hline 24 & 3137.23 & 12.15 & 0.4247 & 197.2 & 340.23 & 1.080 \\
35 & 2955.31 & 7.88 & 0.1832 & 116.1 & 200.31 & 0.268 \\
43 & 3014.08 & 6.72 & 0.1401 & 54.8 & 94.55 & 0.232 \\
69 & 2960.59 & 4.16 & 0.0531 & 24.2 & 41.75 & 0.227 \\
\hline
\end{tabular}

In order to fit the corresponding synergy coefficient $\alpha_{\mathrm{s}}$, we introduced LBM [30-32] simulations under bounce-back boundary conditions. The concrete practice is to compare the gas/water relative permeability curves from the LBM simulations under various $\alpha_{\mathrm{s}}$ with the measured curves by the experiments. The value of $\alpha_{\mathrm{s}}$ was selected based on the average relative errors of gas and water relative permeability curves.

The LBM simulation was with the arbitrary density ratio-pseudo potential model revised by Daniel et al. [33]. To characterize the surface tension between gas and water, the modified form of the pressure tensor proposed by $\mathrm{Hu}$ et al. [34] was utilized. The simulation conditions were set as the same with the tests of the conditions of the percolation experiments: the temperature was 20 degrees and the displacement pressure was $3.22 \mathrm{MPa}$. The three-dimensional lattice model was set to $600 \times 300 \times 300$.

The gas/water relative permeability curves of core $24^{\#}$ and core $69^{\#}$ were obtained by LBM simulations, which were shown in Figures 7 and 8 . Thus it could be seen that the larger the synergetic coefficient $\alpha_{\mathrm{s}}$ was, the larger the seepage resistance of the fluid was.

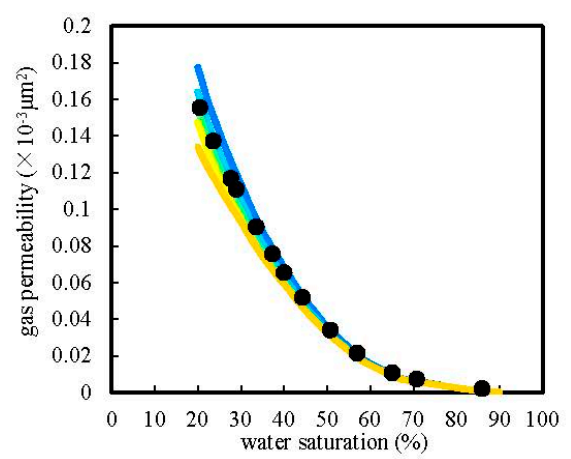

(a)

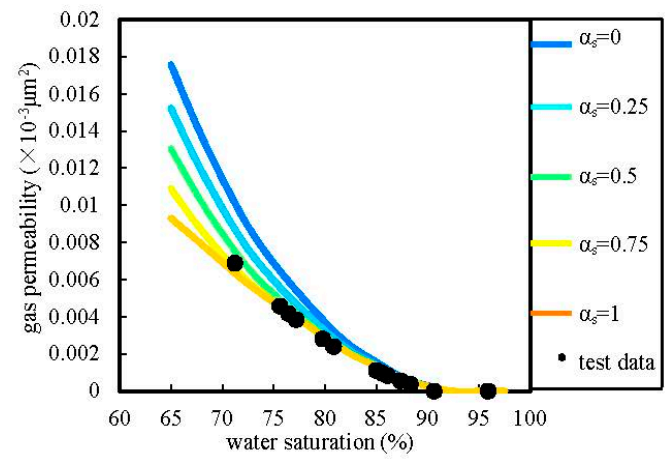

(b)

Figure 7. Gas phase permeability curves of tight sandstone cores $24^{\#}$ and $69^{\#}$. (a) Gas phase permeability curve core $24^{\#}$. (b) Gas phase permeability curve core $69^{\#}$.

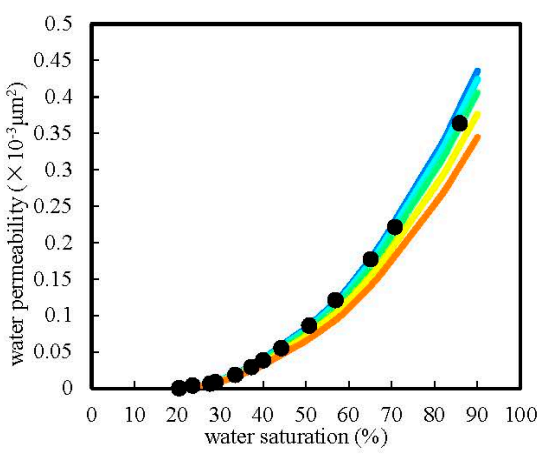

(a)

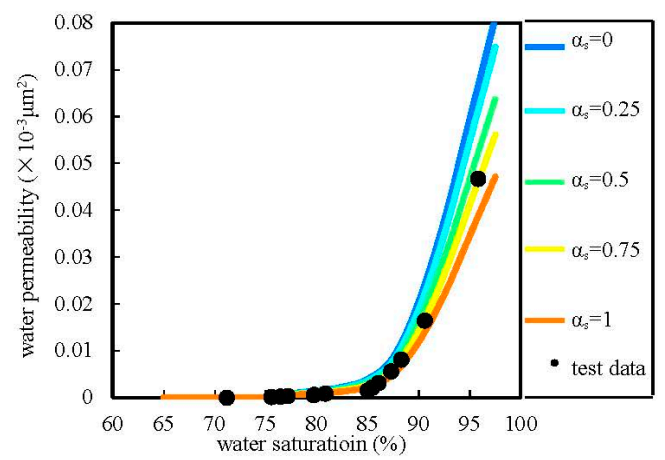

(b)

Figure 8. Water phase permeability curves of tight sandstone cores $24^{\#}$ and $69^{\#}$. (a) Water phase permeability curve core $24^{\#}$. (b) Water phase permeability curve core $69^{\#}$. 
The average relative permeability errors of gas and water under various $\alpha_{\mathrm{s}}$ were shown in Figures 9 and 10. For core $24^{\#}$ and core $69^{\#}$, it could be seen definitely that when the $\alpha_{\mathrm{s}}$ were 0.25 and 0.75 respectively, the average relative permeability errors reached the minimum. That was to say, according to the $\alpha_{\mathrm{s}}$ for the cores above, in terms of the fluid flow capacity, the corresponding simplified physical models were the closest to the tested tight sandstone cores. By the same method, the synergistic coefficients $\alpha_{\mathrm{s}}$ of core $35^{\#}$ and core $43^{\#}$ were 0.62 and 0.47 , respectively.

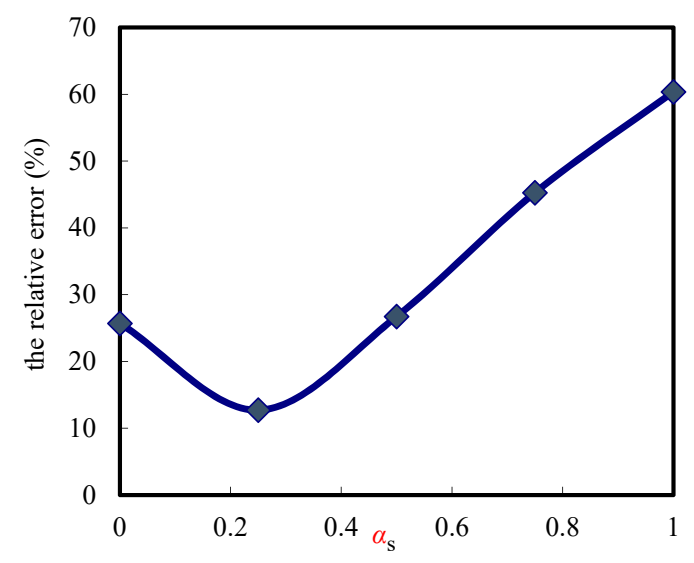

Figure 9. The two-phase permeability average relative errors of core $24^{\#}$.

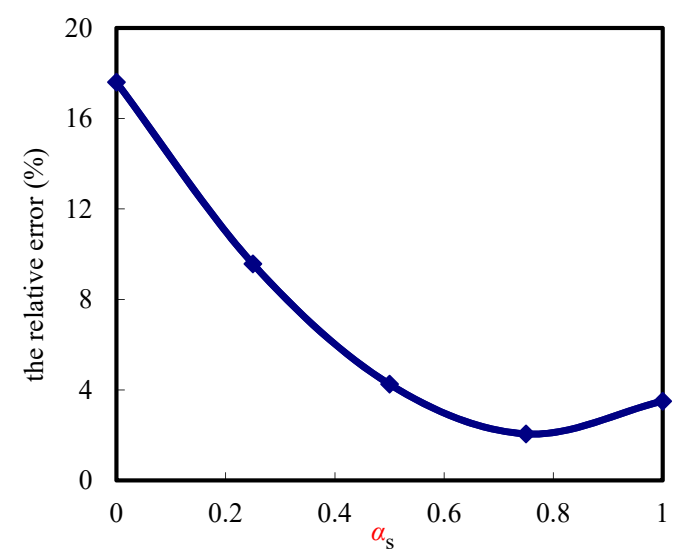

Figure 10. The two-phase permeability average relative errors of core $69^{\#}$.

Among the tight cores above, there are the greatest physical properties difference in core $24^{\#}$ and core $69^{\#}$. As a consequence, we took them as examples to demonstrate our research results. The corresponding three dimensional simplified models of tight core $24^{\#}$ and core $69^{\#}$ were set up by the embedded spheres models which were shown in Figures 11 and 12.

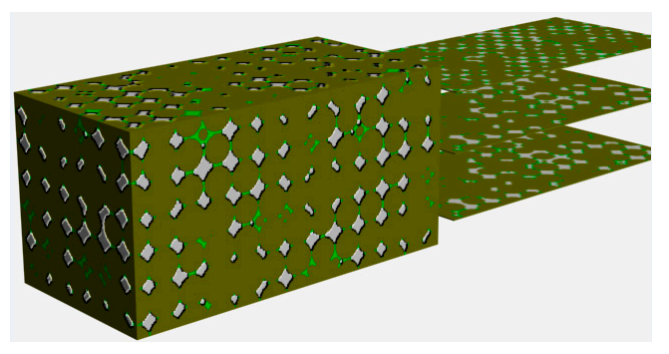

Figure 11. Three-dimensional simplified model diagram of core $24^{\#}\left(\alpha_{\mathrm{s}}=0.25\right)$. 


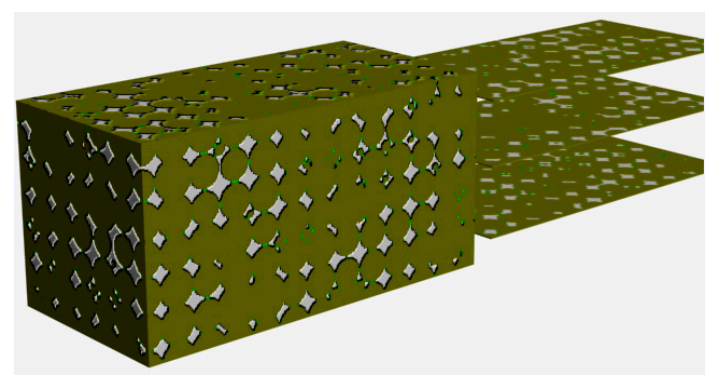

Figure 12. Three-dimensional simplified model diagram of core $69^{\#}\left(\alpha_{\mathrm{s}}=0.75\right)$.

In the figures, the dark green parts are the rock particles, the fresh green parts are the cementation and the white parts are the fluid flow space in the tight porous medium. For core $24^{\#}$, more fresh green parts mean more cementation, in other words, the porosity reduction is mainly caused by cementation for this tight core. On the contrary, it is mainly caused by compaction, such as core $69^{\#}$.

\section{Applications}

\subsection{Seepage Mechanism in Micro Scale}

Compared to the actual tight sandstone reservoirs with higher temperature and pressure, the laboratory experimental conditions show great differences. Furthermore, when the fluid is transferred from the reservoir to the ground, the pore structure and physical properties of tight cores would be different as well. Therefore, it is difficult to accurately characterize the actual flow characteristics of gas and water in tight reservoirs using laboratory experimental results. Applying the LBM simulations, we conducted a comparative study on the gas and water flow laws by the numerical simulation on a micro scale under the laboratory conditions and the actual reservoir conditions.

Taking the simplified model of core $24^{\#}$ as an example, under the experimental conditions the pressure was set to be $1.01 \times 10^{5} \mathrm{~Pa}$, the temperature was $293.15 \mathrm{~K}$, and the displacement pressure gradient was $0.644 \mathrm{MPa} / \mathrm{cm}$. The corresponding interface tension was $0.07197 \mathrm{~N} / \mathrm{m}$, the kinetic viscosity of methane was $0.011 \mathrm{mPa} \cdot \mathrm{s}$, and the kinetic viscosity of water was $0.890 \mathrm{mPa} \cdot \mathrm{s}$ for the simulation. Under actual tight reservoir conditions, the pressure were set to be $300 \times 10^{5} \mathrm{~Pa}$, the temperature was $383.15 \mathrm{~K}$, and the displacement pressure gradient was $0.644 \mathrm{MPa} / \mathrm{cm}$. The corresponding interface tension was $0.03386 \mathrm{~N} / \mathrm{m}$, the kinetic viscosity of methane was $0.022 \mathrm{mPa} \cdot \mathrm{s}$, and the kinetic viscosity of water was $0.259 \mathrm{mPa} \cdot \mathrm{s}$ for the simulation. The three-dimensional lattices were set to be $600 \times 300 \times 300$. The model was fully saturated with water in the initial period and subsequently, gas flooding water was simulated. During the simulation, the fluid seepage laws and the occurrence states under various water saturation conditions were obtained. Suppose that the left cross-section of the lattice model was at the position of $Z=0$. Figure 13 was the distribution of gas and water at different positions $(Z=50,180$ and 230$)$ of core $24^{\#}$ with the porosity of $12.15 \%$ under laboratory experimental conditions. The distribution of gas and water under various water saturation conditions at different positions were shown in Figure 14.

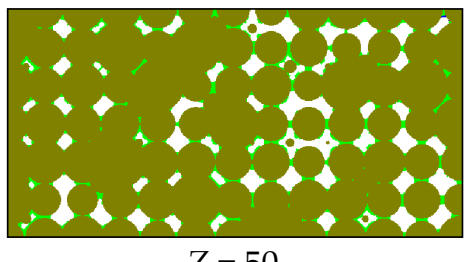

$Z=50$

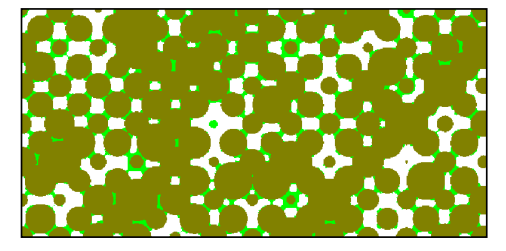

$Z=180$

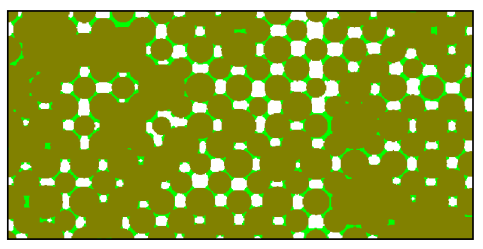

$Z=230$

Figure 13. Different cross sections of the simplified model for the tight sandstone core $24^{\#}$. 

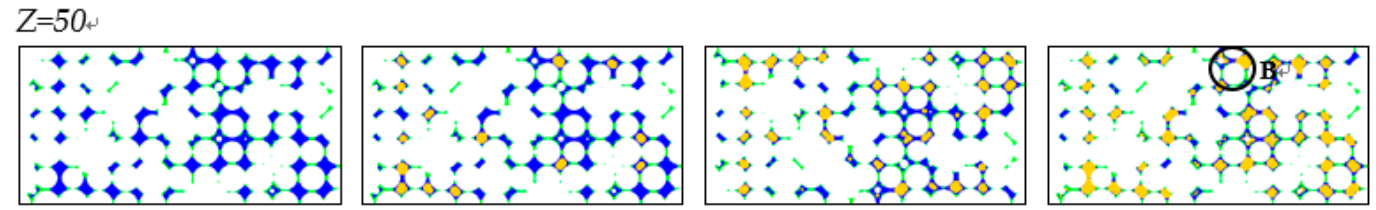

$Z=180$
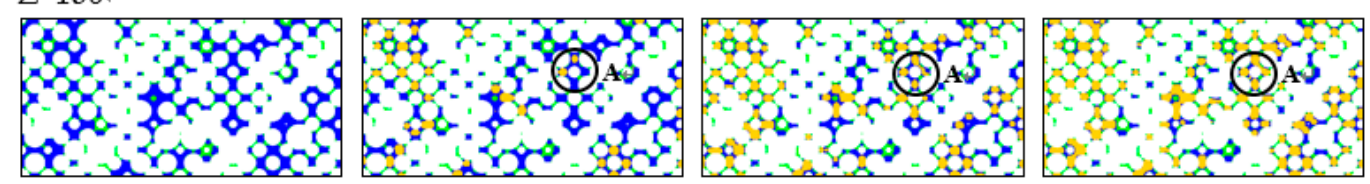

$Z=230$
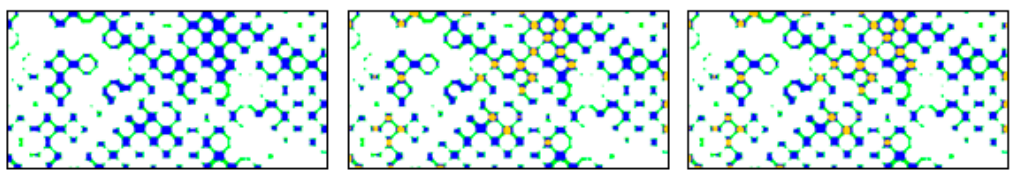

(a) $t=0$ s $S_{w}=100 \%$

(b) $t=1000 \mathrm{~s} S_{w}=63.64 \%$

(c) $t=2000 \mathrm{~s} S_{w}=46.41 \%$

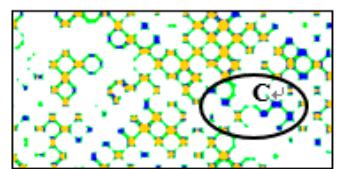

(d) $t=5000 s S_{w}=29.28 \%$

Figure 14. Distribution of gas and water on different cross sections of core $24^{\#}$ under laboratory experimental temperature and pressure conditions.

In the following figures, the rock mineral particles were the white parts. Besides, the blue parts were water phase, the yellow parts were the gas phase and the green parts were the cementation. It was worth noting that the water saturation in the diagrams were the water content of the whole model at that moment. Under high temperature and pressure conditions $\left(p=300 \times 10^{5} \mathrm{~Pa}, T=383.15 \mathrm{~K}\right)$, the distribution of gas and water at different positions $\left(Z=50,180\right.$ and 230) of core $24^{\#}$ with the porosity of $12.15 \%$ are shown in Figure 15.
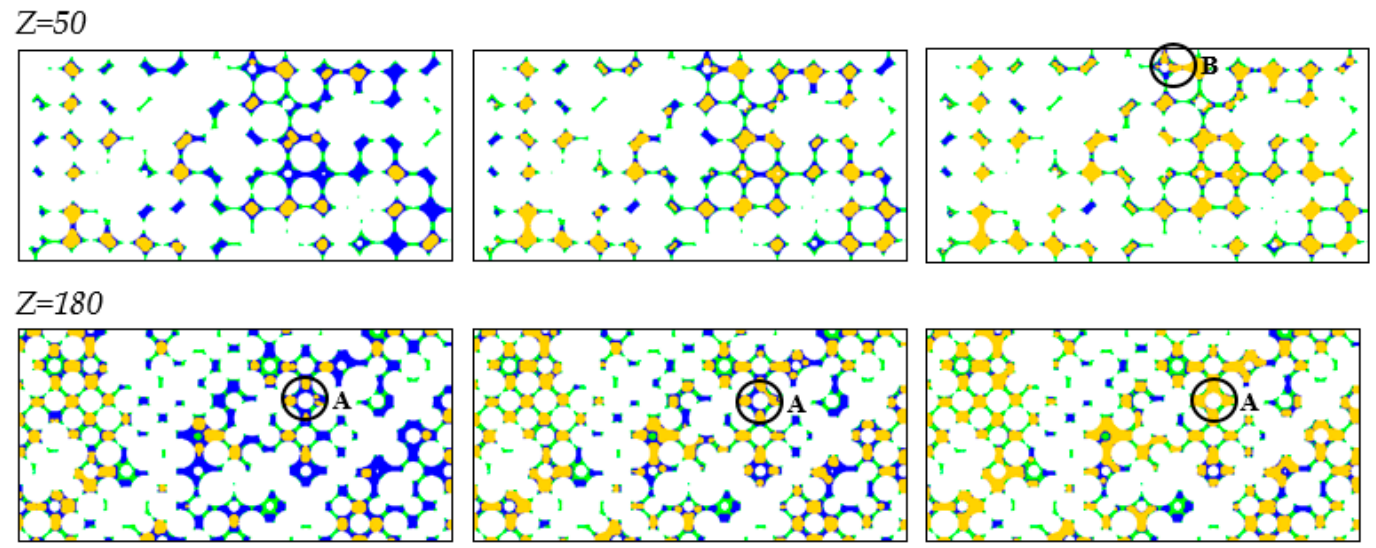

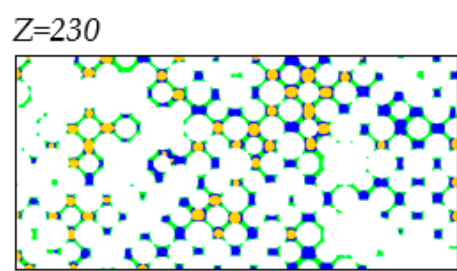

(a) $t=1000 \mathrm{~s} \quad S_{w}=49.18 \%$

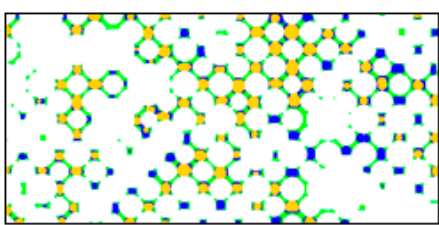

(b) $t=2000 \mathrm{~s} \quad S_{w}=30.52 \%$

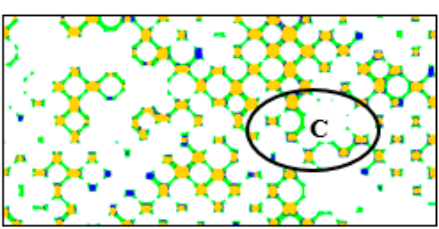

(c) $t=5000 s \quad S_{w}=16.73 \%$

Figure 15. Distribution of gas and water on different cross sections of core $24^{\#}$ under high temperature and pressure conditions.

Comparing Figures 14 and 15, the gas seepage capacity was stronger under actual tight reservoir conditions, namely the high temperature and pressure conditions. Besides, the gas saturation was 
$12.55 \%$ higher than that of the laboratory experimental conditions when the process of gas flooding water entered into the stable state $(t=5000 \mathrm{~s})$. From the figures, as the gas phase was the non-wetting phase, it generally occupied the central parts of the pores and throats while the water phase is usually attached to the rock surface. Next, we take three areas A, B, and C as an example to compare and analyze the fluid flow characteristics under different simulation conditions. From the area A in Figure 14, some of the trapped gas could continue flowing through the small throats as the displacement time is extended, while the phenomenon of cutting-off in the tiny throats was still not changed. In addition, the small gas bubbles were still trapped by the aqueous phase, and unable to flow. In contrast, in Figure 15 the phenomenon of gas being trapped still exists, however, the confined gas in the pores and throats could be broken into several smaller bubbles under the effect of the gas-water interface force and gradually form a continuous flow. This was due to the more intense thermal movement of gas and water molecules under the high temperature and pressure conditions. The displacement pressure generates a continuous disturbance to the interaction potential of gas and water molecules. In order to reduce the interfacial free energy, gas bubbles are broken into smaller gas bubbles which can more easily go through the small throats.

For the area B, it was not hard to find that the cylindrical water phase which was cut off in the throats could not be effectively driven under laboratory experimental conditions. However, under actual tight reservoir conditions, the water phase trapped in the throats which exceeded a certain size could be driven and this phenomenon was more common (as shown in the area C). In addition, when $t$ is equal to $5000 \mathrm{~s}$, it could be found at various positions that the water film bounded on the surface was slightly thinner under high temperature and pressure conditions. This was due to the fact that the diffused double layer between the rock surface and the bounded water would stabilize to a new equilibrium under the higher temperature conditions, namely, the high temperature reduced the electrostatic force. Under the combined impact of the factors mentioned above, the strength of the micro force between the rock surface and the formation water was slightly lower compared to that under laboratory experimental conditions. As a result, the gas phase could drive some of the water column which was originally stuck in the throats, so that the water saturation in actual tight reservoirs was lower.

\subsection{Gas and Water Relative Permeability Curves under Various Conditions}

Based on the LB method, we programmed a computer program which took the wall surface of the simplified model as the boundary conditions and conducted the simulations of the gas/water seepage laws under laboratory experimental conditions and actual tight reservoir conditions respectively. The relative permeability curves of gas and water under various simulation conditions of four tight gas cores are shown in Figures 16-19. The simplified physical model and the simulating results by LB method were also verified with the experimental results under indoor experimental conditions, and these two are consistent.

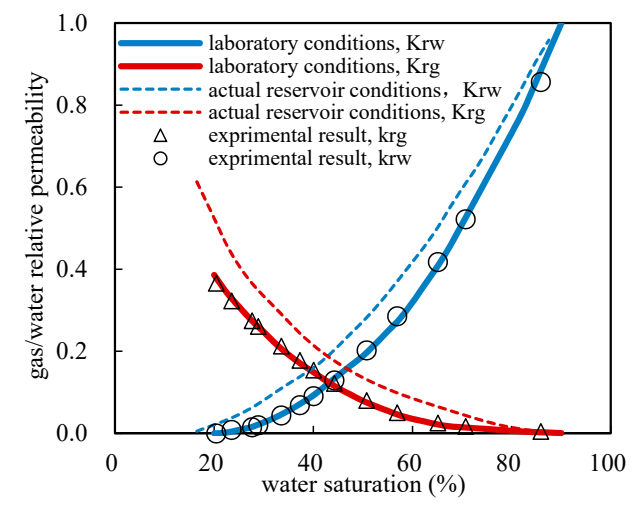

Figure 16. Gas/water relative permeability curves of core $24^{\#}$. 


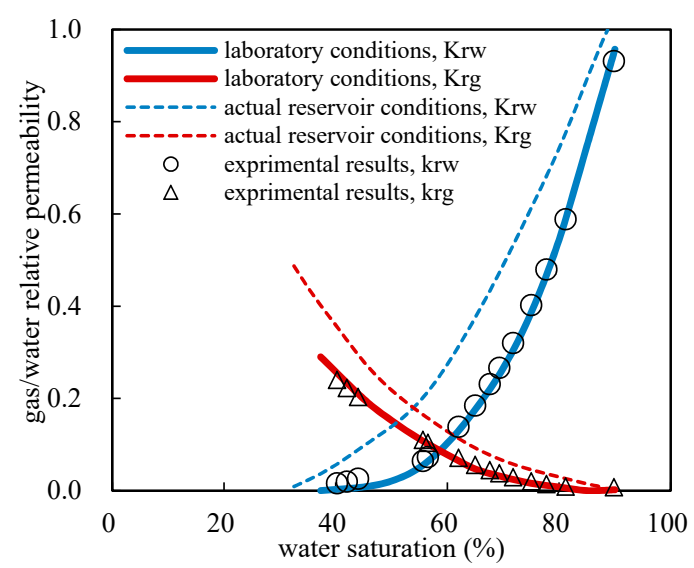

Figure 17. Gas/water relative permeability curves of core $35^{\# .}$

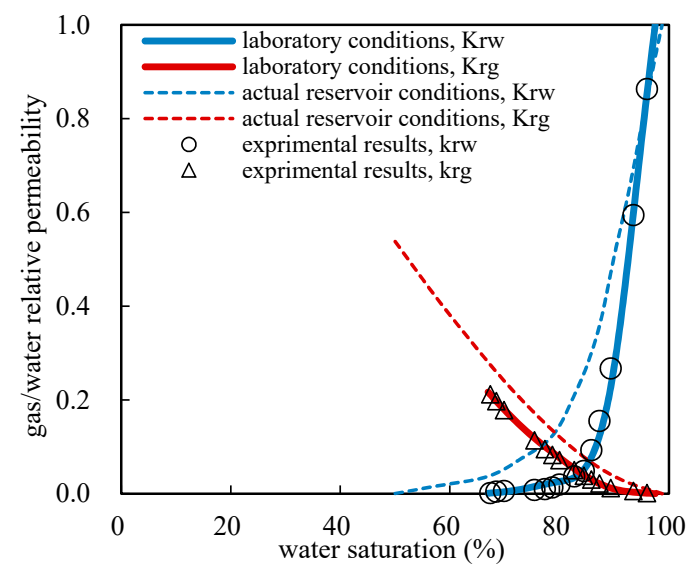

Figure 18. Gas/water relative permeability curves of core $43^{\#}$.

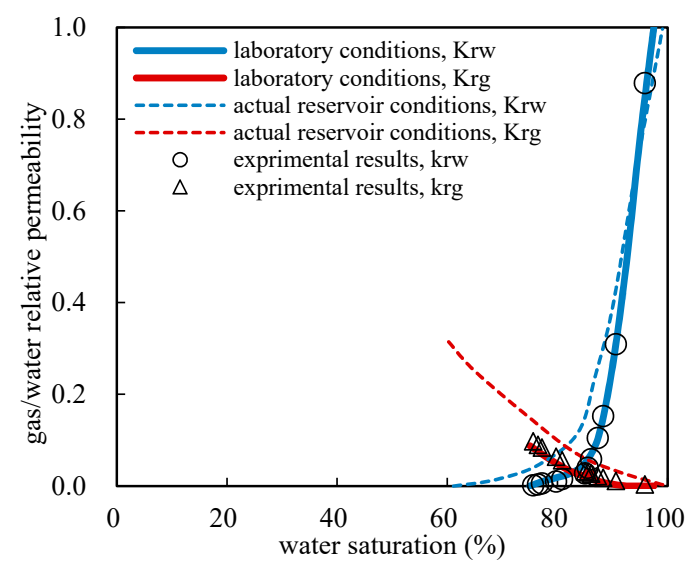

Figure 19. Gas/water relative permeability curves of core $69^{\# .}$

Comparing the gas/water relative permeability curves under various conditions, the following phenomena are observed under high temperature and pressure simulation conditions: the irreducible water saturation decreases and the region of gas and water co-seepage is larger; the isotonic point of gas and water moved to the left side, and the poorer the physical properties of the tight cores were, the greater the amplitude of the left shift was; under the same water saturation conditions, the relative permeability of gas phase was generally higher. The phenomena above also proved the conclusion 
that both gas and water had stronger percolation capacity in the actual tight gas reservoirs compared to the laboratory experimental conditions.

From Figure 20, core $24^{\#}$ had the best physical properties that its irreducible water saturation reduced by $4 \%$ under high temperature and pressure simulating conditions. While core $69^{\#}$ had the poorest physical properties where its irreducible water saturation reduced by $16.9 \%$. Furthermore, the lower the permeability of the tight core was, the greater the differences were. This was because the lower the porosity and permeability of the tight cores were, the more significant the influence of the rock surface on the capacity of fluid flow was. Under high temperature and high pressure conditions, the fluid could more easily flow due to the effect of the electrostatic force between the wall surface and the fluid. Moreover, the thermal motions of gas and water molecules were more intense, thus the interfacial tension between gas and water was also decreased, so that the swept volume was relatively larger during the displacement. Consequently, the fluid had stronger seepage capacity under actual tight gas reservoir conditions.

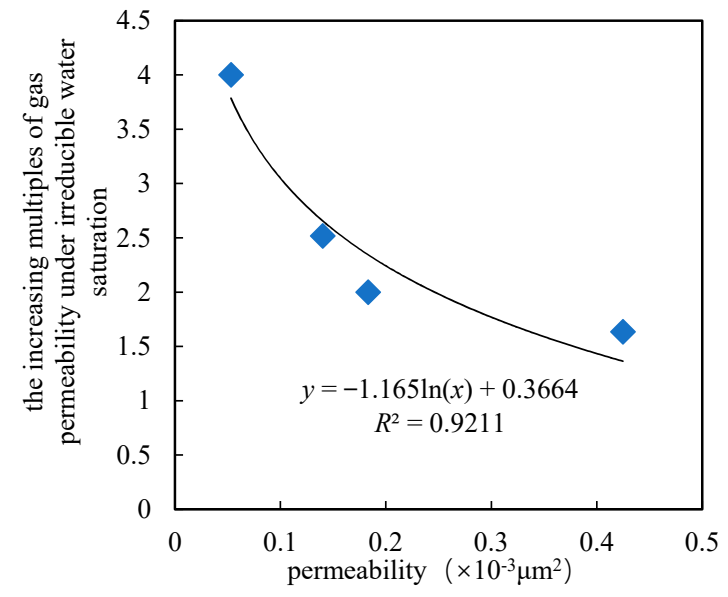

Figure 20. The increasing multiples of the gas permeability versus the irreducible water saturation under high temperature and pressure conditions.

\section{Conclusions}

A simplified physical model construction method for tight sandstone gas reservoirs was established based on some geological origin features, namely the sedimentary compaction and clay mineral-cementation. The synergistic coefficient was proposed to embody the coupling effects of the above two factors, and its fitting method was also established. The porosity, permeability and the gas/water relative permeability curves were equivalent and consistent comparing the simplified model with the actual tight core. This model provides a good theoretical reference and a new direction for the research on microscopic seepage mechanism of tight sandstone reservoirs. The following systematic insights were obtained by applying the simplified physical model to simulate the process of gas flooding water under different conditions:

(1) During the displacement, as the interfacial tension between gas and water gets lower and the swept volume gets larger, both gas and water have stronger seepage ability under actual tight sandstone gas reservoir conditions.

(2) Under high temperature and high pressure, the diffused double layer between the rock surface and the formation water would be stabilized in a new equilibrium state where the water film adhered to the rock surface was poorer in stability. Consequently, the bound water film gets thinner.

(3) Compared with the simulation results under laboratory temperature and pressure conditions, some gas bubbles confined at the narrow originally were broken into smaller gas bubbles under 
actual reservoir temperature and pressure conditions which reduced their interfacial free energy, therefore, more gas continuous flow occurred.

(4) The irreducible water saturation got decreased and the co-seepage region of gas and water got larger under actual tight sandstone gas reservoir conditions. Moreover, the lower the permeability of the tight core was, the greater the differences would be.

Author Contributions: F.W. and Y.L. developed the simplified physical model and performed the simulations reported in this paper; C.H. designed the LBM code adopted for the numerical simulations; A.S. and S.L. contributed to the results analysis and post-processing; B.C. wrote the paper. All authors reviewed the manuscript.

Funding: This research was supported by the National Science and Technology Major Project of China (2016ZX05023005) and RIPED (Research Institute of Petroleum Exploration\& Development) at Langfang for financial support.

Conflicts of Interest: The authors declare no conflict of interest.

\section{Nomenclature}

$\begin{array}{ll}r & \text { the radius of rock particle, nm } \\ r_{\min } & \text { the minimum radius of rock particles, } \mathrm{nm} \\ r_{\max } & \text { the maximum radius of rock particles, } \mathrm{nm} \\ f(r) & \text { the particle sizes distribution function of any tight sandstone particles } \\ \sigma & \text { the peak value on the Rayleigh frequency distribution curve } \\ r_{\mathrm{C}} & \text { the sealing radius of the clay minerals, } \mathrm{nm} \\ r_{1}, r_{2} & \text { the minimum and the maximum radius of rock particles in any particle sizes range, } \mathrm{nm} \\ g\left(r_{1}, r_{2}\right) & \text { the probability density of the Rayleigh distribution function of particle sizes in the range of } \mathrm{r}_{1} \\ R & \text { the sphere radius of the ideal physical model, nm } \\ \bar{r} & \text { the equivalent radius of the flow space in the ideal physical model, nm } \\ \phi_{\mathrm{S}} & \text { the porosity reduction caused by compaction } \\ \phi_{\mathrm{c}} & \text { the porosity reduction caused by cementation } \\ \alpha_{\mathrm{S}} & \text { the synergy coefficient } \\ p & \text { pressure, Pa } \\ T & \text { reservoir temperature, } \mathrm{K} \\ Z & \text { the cross-section position of the lattice model from the left side } \\ S_{\mathrm{W}} & \text { water saturation, } \%\end{array}$

\section{References}

1. Muccino, J.C.; Gray, W.G.; Lin, A.F. Toward an improved understanding of multiphase flow in porous media. Rev. Geophys. 1998, 36, 401-422. [CrossRef]

2. Porter, M.L.; Schaap, M.G.; Wildenschild, D. Lattice-Boltzmann simulations of the capillary pressure-saturation-interfacial area relationship for porous media. Adv. Water Resour. 2009, 32, 1632-1640. [CrossRef]

3. Zuo, L.H.; Weijermars, R. Rules for Flight Paths and Time of Flight for Flows in Porous Media with Heterogeneous Permeability and Porosity. Geofluids 2018, 3, 1-18. [CrossRef]

4. Li, Y.W.; Jia, D.; Liu, J.; Fu, C.K.; Ai, C. The calculation method based on the equivalent continuum for the fracture initiation pressure of fracturing of coalbed methane well. J. Petrol. Sci. Eng. 2016, 146, 909-920. [CrossRef]

5. Liu, Y.K.; Wang, F.J.; Tang, H.M.; Liang, S. Well type and pattern optimization method based on fine numerical simulation in coal-bed methane reservoir. Environ. Earth Sci. 2015, 73, 5877-5890. [CrossRef]

6. Kim, C.; Jang, H.; Lee, Y. Diffusion characteristics of nanoscale gas flow in shale matrix from Haenam basin, Korea. Environ. Earth Sci. 2016, 75, 1-8. [CrossRef]

7. Brown, S.R. Fluid flow through rock joints: The effect of surface roughness. J. Geophys. Res. Solid Earth 1987, 92, 1337-1347. [CrossRef]

8. Zhao, Z.; Li, B.; Jiang, Y. Effects of Fracture Surface Roughness on Macroscopic Fluid Flow and Solute Transport in Fracture Networks. Rock Mech. Rock Eng. 2014, 47, 2279-2286. [CrossRef] 
9. Yu, W.; Sepehrnoori, K.; Patzek, T.W. Evaluation of Gas Adsorption in Marcellus Shale. In Proceedings of the SPE Technical Conference and Exhibition, Amsterdam, The Netherlands, 27-29 October 2014.

10. Li, Y.W.; Zuo, L.; Yu, W.; Chen, Y.G. A Fully Three Dimensional Semianalytical Model for Shale Gas Reservoirs with Hydraulic Fractures. Energies 2018, 11, 436. [CrossRef]

11. Zhao, H.Q.; Macdonald, I.F.; Kwiecien, M.J. Multi-Orientation Scanning: A Necessity in the Identification of Pore Necks in Porous Media by 3-D Computer Reconstruction from Serial Section Data. J. Colloid Interface Sci. 1994, 162, 390-401. [CrossRef]

12. Shin, H.; Lindquist, W.B.; Sahagian, D.L. Analysis of the vesicular structure of basalts. Comput. Geosci. 2005, 31, 473-487. [CrossRef]

13. Al-Kharusi, A.S.; Blunt, M.J. Network extraction from sandstone and carbonate pore space images. J. Petrol. Sci. Eng. 2007, 56, 219-231. [CrossRef]

14. Martys, N.S.; Chen, H. Simulation of multicomponent fluids in complex three-dimensional geometries by the lattice Boltzmann method. Phys. Rev. E 1996, 53, 743. [CrossRef]

15. Ahrenholz, B.; Tölke, J.; Lehmann, P. Prediction of capillary hysteresis in a porous material using lattice-Boltzmann methods and comparison to experimental data and a morphological pore network model. Adv. Water Resour. 2008, 31, 1151-1173. [CrossRef]

16. Joekar-Niasar, V.; Prodanović, M.; Wildenschild, D. Network model investigation of interfacial area, capillary pressure and saturation relationships in granular porous media. Water Resour. Res. 2010, 17, 370-374. [CrossRef]

17. Kim, K.N.; Kang, J.H.; Sang, G.L. Lattice Boltzmann simulation of liquid water transport in microporous and gas diffusion layers of polymer electrolyte membrane fuel cells. J. Power Sources 2015, 278, $703-717$. [CrossRef]

18. Lei, Z.; Yao, J.; Hai, S. Lattice Boltzmann simulation of gas desorption and diffusion based on digital core technology. Acta Petrol. Sin. 2015, 36, 361-365.

19. Ji, L.; Lin, M.; Jiang, W. An Improved Method for Reconstructing the Digital Core Model of Heterogeneous Porous Media. Transp. Porous Media 2018, 121, 389-406. [CrossRef]

20. Spencer, A.J. A theory kinematics or ideal soils under plane strain conditions. J. Mech. Phys. Solids. 1976, 43, 49-53. [CrossRef]

21. Morrison, H.L.; Richmond, O. Application of Spencer's Ideal Soil Model to Granular Materials Flow. J. Appl. Mech. 1976, 43, 49-53. [CrossRef]

22. Zou, C.N.; Yang, Z.; Tao, S.Z.; Li, W.; Wu, S.T.; Hou, L.H.; Zhu, R.K.; Yuan, X.J.; Wang, L.; Gao, X.H.; et al. Nano-hydrocarbon and the accumulation in coexisting source and reservoir. Petrol. Explor. Dev. 2012, 39, 13-26. [CrossRef]

23. Wang, Z.; Guo, Y.; Wang, M. Permeability of high-Kn real gas flow in shale and production prediction by pore-scale modeling. J. Nat. Gas Sci. Eng. 2016, 28, 328-337. [CrossRef]

24. Pan, J.G.; Wang, H.B.; Li, C.; Zhao, J.G. Effect of pore structure on seismic rock-physics characteristics of dense carbonates. Appl. Geophys. 2015, 12, 1-10. [CrossRef]

25. Zhu, H.H.; Zhong, D.K.; Zhang, Y.X.; Sun, H.T.; Du, B.Q.; Meng, H.; Zhang, C.W.; Yang, Z. Pore types and controlling factors on porosity and permeability of Upper Triassic Xujiahe tight sandstone reservoir in Southern Sichuan Basin. Oil Gas Geol. 2014, 35, 65-76.

26. Mcbride, E.F. Quartz cement in sandstones: A review. Earth-Sci. Rev. 1989, 26, 69-112. [CrossRef]

27. De, V.J.; Poesen, J.; Bazzoffi, P.; Rompaey, A.V.; Verstraeten, G. Predicting catchment sediment yield in Mediterranean environments: The importance of sediment sources and connectivity in Italian drainage basins. Earth Surf. Process. Landf. 2010, 31, 1017-1034.

28. Li, Y.; Jia, A.; He, D. Control factors on the formation of effective reservoirs in tight sands: Examples from Guang'an and Sulige gasfields. Acta Petrol. Sin. 2013, 34, 71-82.

29. Jing, Z.; Shuangwen, L.I.; Wang, S.; Ji, H.S.; Rao, M. Deep diagenetic evolution and formation mechanisms of secondary porosity in Qinan ramp, Qikou sag. J. China Univ. Min. Technol. 2014, 43, 1070-1077.

30. Ren, J.J.; Guo, P.; Guo, Z.L.; Wang, Z. A Lattice Boltzmann Model for Simulating Gas Flow in Kerogen Pores. Transp. Porous Media 2015, 106, 285-301. [CrossRef]

31. Zhang, X.L.; Xiao, L.Z.; Guo, L.; Xie, Q.M. Investigation of shale gas microflow with the Lattice Boltzmann method. Petrol. Sci. 2015, 12, 96-103. [CrossRef] 
32. Krastev, V.; Falcucci, G. Simulating Engineering Flows through Complex Porous Media via the Lattice Boltzmann Method. Energies 2018, 11, 715. [CrossRef]

33. Daniel, L.B.; Luo, K.H. Improved forcing scheme in pseudopotential lattice Boltzmann methods for multiphase flow at arbitrarily high density ratios. Phys. Rev. E 2015, 91, 1-12.

34. Hu, A.J.; Li, L.; Chen, S.; Liao, Q.; Zeng, J. On equations of state in pseudo-potential multiphase lattice Boltzmann model with large density ratio. Int. J. Heat Mass Transf. 2013, 67, 159-163. [CrossRef] 